\title{
PISA: el precio pedagógico de una evaluación internacional
}

\author{
PISA: the Pedagogical Price of an International Evaluation
}

Roberto Sanz Ponce (*) https://orcid.org/0000-0003-1147-743X

Ángela Serrano Sarmiento (*) https://orcid.org/0000-0003-4577-5785

Aurelio González Bertolín (*) https://orcid.org/0000-0003-3167-297X

(*) Universidad Católica de Valencia

(Recibido: 18 de octubre de 2018; Aceptado para su publicación: 11 de febrero de 2019)

Cómo citar: Sanz, R., Serrano, A. y González, A. (2020). PISA: el precio pedagógico de una evaluación internacional. Revista Electrónica de Investigación Educativa, 22, e22, 1-13. https://doi.org/10.24320/redie.2020.22.e22.2673

\section{Resumen}

El objetivo del artículo es comprobar si el "mal uso" de los resultados PISA tiene consecuencias perversas para el sistema educativo y sus docentes. Para ello se empleó la metodología hermenéutica mediante la reflexión de indicadores extraídos del análisis de datos. Entre los resultados obtenidos se observó una mejora constante del estudiantado español en Matemáticas, Ciencias y Comprensión lectora en las diferentes evaluaciones 2006-2016, pero también se detectó cómo el sistema educativo se ha transformado y adaptado a las necesidades y solicitudes de PISA, independientemente de las necesidades de los alumnos. Como conclusiones se evidencia que PISA orienta las políticas educativas; que los medios y los políticos distorsionan la realidad de los resultados y sitúan en la diana al docente; que la creatividad, innovación, diversidad, educación en valores y desarrollo de capacidades quedan relegadas; que el currículum y el horario priorizan ciertas asignaturas y que la equidad se resquebraja, generando fractura social.

Palabras clave: PISA, docentes, calidad de la educación, sistema educativo, evaluación.

\begin{abstract}
This article aims to determine whether the "misuse" of PISA results has adverse impacts on the education system and its teachers. A hermeneutic methodology was employed, which consisted in a reflection on indicators identified from data analysis. The results observed included a continuous improvement among Spanish students in mathematics, science, and reading comprehension in different evaluations from 2006 to 2016, but the research also identified how the education system has changed and adapted based on the needs and requests of PISA, regardless of the needs of the students themselves. In conclusion, this study shows that PISA informs education policy; the media and politicians distort the reality of results while targeting teachers; creativity, innovation, diversity, values education, and capacity building are being left behind; the school curriculum and schedules prioritize certain subjects; and equity is falling apart, leading to social divisions.
\end{abstract}

Keywords: PISA, teachers, quality of education, education system, evaluation. 


\section{Introducción}

Disponer de un sistema educativo que proporcione una enseñanza de calidad constituye una aspiración universal. Nadie mínimamente juicioso afirmaría que no desea una educación de calidad para sus hijos, comunidad o país. En 1988, la Sociedad Española de Pedagogía (SEP) manifestaba que "la calidad y la excelencia son metas deseables de la educación [...] resulta difícil imaginar una escuela o una organización satisfechas en su mediocridad" (SEP, 1988, p. 7).

Lo enunciado pudiera parecer una obviedad, sin embargo, constituye una misión mucho más difícil precisar qué se entiende por calidad educativa y qué características debiera tener un sistema educativo de calidad. Así pues, el término calidad educativa abarca todo el espacio ideológico, por lo que genera controversia. La Organización para la Cooperación y el Desarrollo Económicos (OCDE, 1991) lo reconoce cuando afirma que el concepto calidad educativa significa cosas diferentes para distintos observadores, ya que no todos comparten problemas, objetivos y prioridades.

La calidad como prioridad de los sistemas educativos aparece en las agencias internacionales en 1983, cuando en la conferencia de la OCDE los ministros de educación de los países miembros manifestaron que la consecución de la calidad educativa constituiría una prioridad, aunque ésta posteriormente sólo se ha identificado con la productividad. La idea predominante es que la calidad de la educación viene determinada por el rendimiento escolar de los alumnos (Puelles, 2009).

Con ese espíritu se inició en 1997 el Programa para la Evaluación Internacional de los Alumnos (PISA), auspiciado por la OCDE para analizar el rendimiento escolar de los estudiantes de distintos países al final del período de escolarización obligatoria. Planteaba la necesidad de contar con datos comparables para establecer indicadores de evaluación.

Estas pruebas se organizan de manera conjunta por los países miembros de la oCDE y cuentan cada vez con mayor número de asociados. En el caso de España, cada Comunidad Autónoma, en ejercicio de sus competencias, decide su participación. La evaluación se realiza cada tres años (2000, 2003, 2006, 2009, 2012 y 2015). En el año 2000 participaron 32 países, entre ellos España; en la segunda edición 42, uniéndose las Comunidades Autónomas de Castilla-León, País Vasco y Cataluña. En 2006 ya eran 57 y, a las tres anteriores, se añadieron Galicia, Asturias, La Rioja, Aragón y Navarra. En 2009 participaron 64 países y 14 Comunidades Autónomas. En las pruebas celebradas en 2012 fueron evaluados un poco más de 500000 alumnos de 65 países. Y en la última edición (2015), 540000 estudiantes de 72 países. Además todas las Comunidades Autónomas, según la organización, han ampliado su muestra de participación para recabar datos representativos que sean comparables a nivel internacional.

Según los organizadores los objetivos del proyecto son:

a) Extraer conclusiones, a partir de los resultados, que permitan identificar fortalezas y debilidades de los sistemas educativos para orientar sus políticas.

b) Centrarse en competencias: adquisición de destrezas y habilidades que pudieran aplicarse por parte del alumnado evaluado a situaciones relativamente distintas a las contempladas en los contenidos escolares.

c) Identificar otros factores vinculados al estudiante: sensibilidades, motivaciones, sentimientos y estrategias de aprendizaje.

d) Situar el rendimiento del alumno en relación con las características socioeconómicas y culturales de las familias, entorno y tipología del centro educativo al que pertenece. 
Así pues, PISA constituye un estudio comparado, internacional, periódico y continuo (Turner, 2006) sobre las competencias adquiridas por los estudiantes al finalizar su escolarización obligatoria, si bien se toma como referencia los 15 años de edad, con el fin de unificar los posibles desajustes sobre la duración exacta del tramo obligatorio en los distintos sistemas educativos. Consta de una prueba cognitiva, de dos horas de duración, sobre tres dominios de aprendizaje: comprensión lectora, matemáticas y ciencias. Cada tres años la prueba se centra, predominantemente, en uno de ellos (en un porcentaje aproximado del 65\%). Los ejercicios combinan preguntas de respuesta alternativa, concreta o directa, con otras que admitan una respuesta parcialmente correcta. Asimismo, los estudiantes cumplimentan un cuestionario complementario, de 30 minutos aproximadamente, sobre sus características personales y familiares, opiniones, preferencias e intereses.

Hasta 2006 los ejercicios consistían sólo en pruebas de papel y lápiz. En 2009 se incorporó un ejercicio de lectura en formato digital y en 2015 las pruebas cognitivas y los cuestionarios de contexto fueron exclusivamente en formato digital. Conviene señalar que PISA no pretende evaluar objetivos o contenidos académicos; es decir, lo que los alumnos han aprendido en la escuela, sino su capacidad de movilizarlos y aplicarlos en contextos distintos. "Puede afirmarse que más que la comprensión lectora, las matemáticas y las ciencias, PISA pretende evaluar la competencia lectora, la competencia matemática y la competencia científica" (Tiana, 2009a, p. 45). En definitiva, las destrezas y habilidades adquiridas y asimiladas que permitan a los jóvenes desenvolverse en espacios y situaciones propias del mundo adulto.

Además, los responsables de los centros educativos realizan un cuestionario complementario sobre las características del centro y su entorno, con la intención de extraer conclusiones sobre la relación entre los resultados y el contexto familiar y escolar.

Por tanto, además del rendimiento académico se miden también aspectos como la tipología y organización de los centros educativos, la cualificación de los padres, las dificultades de aprendizaje o el porcentaje de alumnos inmigrantes (Cárdenas y Huertas, 2013). Así pues, se puede afirmar que además de medir los resultados en términos de adquisición de competencias, aporta abundantes datos sobre factores de equidad -relación entre recursos, resultados e igualdad de oportunidades-, así como de ingresos familiares, nivel educativo y cualificación profesional de los padres, estructura y origen cultural de las familias, titularidad y organización de los centros educativos y procedimientos educativos puestos en práctica por el profesorado.

Los propios evaluadores han señalado la importancia de considerar cuatro ámbitos para la adecuada valoración del rendimiento escolar: el nivel de los resultados de aprendizaje, la relación de estos con los indicadores de bienestar social, igualdad de oportunidades y eficacia de los procesos. Por tanto, "la principal aportación de PISA a la evaluación de resultados consiste en combinar de manera equilibrada la valoración de la calidad de la educación, medida a través del logro de ciertas competencias, con la equidad de su distribución" (Tiana, 2009a, p. 54). Y no sólo eso, los Informes han aportado evidencias de que algunos sistemas educativos son capaces de contrarrestar con mayor eficacia la incidencia de estos factores en los resultados obtenidos.

Por tanto, algo que también pone de manifiesto PISA es la posibilidad de aunar equidad y calidad, "se pueden obtener buenos rendimientos para todos los alumnos sin disminuir el de los mejores y sin recurrir a la selección o a la exclusión de ninguno de ellos" (Puelles, 2009, p. 36). PISA evidenció que algunos de los sistemas con mejores resultados son también los más equitativos; países como Finlandia, Canadá, Dinamarca, Hong-Kong y Macao lograron buenos resultados en estudiantes de diferentes niveles socioeconómicos (OCDE, 2015).

En dicho Informe (OCDE, 2015), que se centró en la competencia científica, España en su conjunto, según el Instituto Nacional de Evaluación Educativa (INEE, 2016) obtuvo una puntuación media en lectura (496), ligeramente superior a la del promedio de la OCDE (493) y al de la Unión Europea (494). En matemáticas (486), por debajo de la OCDE y de la Unión Europea. Hay que precisar que en matemáticas España tiene una proporción de alumnos rezagados similar al de la Unión Europea (22\%) y menor al de la OCDE (23\%). Sin embargo, el porcentaje de alumnos excelentes es del 7\%, inferior al de la OCDE (10\%) y al de la Unión Europea (11\%). En ciencias, se sitúa en el mismo promedio de la OCDE (493) y ligeramente por debajo del 
de la Unión Europea. Aunque, en este caso España presenta una proporción de alumnos rezagados (18\%) sensiblemente menor al de la OCDE (22\%) y al de la Unión Europea (21\%). Por el contrario, el porcentaje de alumnos excelentes (5\%) es inferior al de ambas organizaciones (8\%).

En términos generales se trata de unos resultados que mejoran sensiblemente los obtenidos en evaluaciones precedentes. No obstante, parecen reafirmar las características del país: se sitúa ligeramente por debajo de los países que obtienen mejores resultados; con un porcentaje bajo de alumnos de nivel alto o excelentes; y mantiene buena posición con respecto a los indicadores de equidad.

La repercusión mediática de los resultados PISA puede calificarse de inusitada, máxime teniendo en cuenta que se trata de un tema educativo. La información se centra en destacar los ranking de los países y regiones, mencionando los más destacados, identificando los rezagados y, en el caso concreto de España, subrayando el bajo nivel obtenido por los estudiantes (Luzón y Torres, 2013; Tiana, 2009b). La repercusión en la opinión pública de las abundantes informaciones y opiniones de trazo grueso en torno a los resultados no es cuestión baladí, habida cuenta que PISA constituye el programa de evaluación más amplio y es, sin duda, la empresa más influyente a la hora de orientar las reformas y políticas educativas de los distintos países (Lundgren, 2013; Marchesi y Martínez, 2006; Tiana, 2009a; Tiana, 2009b; Puelles, 2009), y así se pone de manifiesto en el caso de España.

La Ley Orgánica de la Mejora de la Calidad de la Educación (LOMCE, 2013) constituye un potente cambio de concepción de las prioridades y objetivos del sistema educativo, pasando de un modelo en el que la calidad de la educación se mide por los recursos puestos al servicio del sistema: inversión, centros, profesores y medios materiales, a otro donde los resultados también cuentan. O mejor dicho, lo que cuenta son los resultados.

Disminuir el fracaso escolar, aumentar la empleabilidad, mejorar los resultados en las evaluaciones internacionales, cohesionar los resultados entre comunidades, fomentar la especialización y autonomía del centro, impulsar las TIC y el plurilingüismo, así como potenciar la orientación, la tutoría y el acompañamiento a los estudiantes, aparecen como objetivos en la LOMCE.

En ese sentido, la mayoría de las novedades que incorpora dicha ley están orientadas a la mejora de los resultados académicos: evaluación externa, diversificación de trayectorias, potenciación de materias instrumentales, planes de refuerzo, fortalecimiento de la función directiva o la revitalización de las enseñanzas de Formación Profesional. En concreto, se puede afirmar que la mayoría de las novedades prescritas en el texto legal parecen claramente orientadas a mejorar los resultados en PISA.

Así pues, cuando se afirma que la educación se encuentra en "crisis" y que no responde a las demandas sociales, convendría hacer una reflexión de trazo fino con relación a otros aspectos, tanto económicos como de inversión de coste humano.

\section{Método}

Se parte de la hipótesis de que el mal uso de los resultados de PISA tiene consecuencias perniciosas para la finalidad del sistema educativo y para el desarrollo del quehacer docente. Por ello, el objetivo establecido es confirmar dicha hipótesis y enumerar y describir cuáles son y cómo afectan esos efectos perniciosos en la calidad de la educación y del proceso de enseñanza-aprendizaje.

Para conseguir dicho objetivo se tomaron los datos aportados por PISA para los alumnos españoles en las evaluaciones de 2006-2016; analizando la literatura existente en torno a esta problemática para, por último, utilizar una metodología de tipo hermenéutico que permita interpretar -no sólo explicar- de manera crítica y contextualizada estos resultados. En este sentido, frente a los fríos datos arrojados por PISA, se busca presentar un relato que dé sentido y comprensión a esta realidad. Como decía Gadamer (1977, p. 585), "lo que no logra la herramienta del método tiene que conseguirlo y puede realmente hacerlo, una disciplina del preguntar y el investigar, que garantice la verdad." 


\section{Análisis comparativo de los resultados en PISA: la realidad española en 10 años}

PISA manifiesta la importancia de medir logros y resultados educativos (Jornet, 2016; Martínez-Rizo, 2016). Sin embargo, recabar indicadores de logro educativo dentro del marco general de indicadores que trabaja habitualmente la OCDE supone una dificultad en la significancia de dichos indicadores, especialmente en el diseño de los formatos de evaluación (Jornet, 2016):

La realidad es que, tal como señalan diversos autores, la definición de competencias medidas en el proyecto están orientadas a un tipo de sociedad en la que los ingenieros y tecnólogos constituyan finalmente la élite que permita evolucionar económicamente a un país en un mismo modelo de desarrollo (Martínez-Rizo, 2016). Las necesidades de formación para desarrollarse personalmente de manera adecuada en un espacio geopolítico y social determinado, no tienen por qué ser los mismos en todos los países. De ahí la necesidad de que se reconsideren las competencias a evaluar y se analicen en su definición respecto a las características locales, que no son sólo las que se definen en un diseño curricular nacional. (p. 9).

Con ello, los resultados obtenidos por España en los últimos 10 años suponen un logro:

Tabla I. Resultados en Comprensión lectora (2006-2016)

\begin{tabular}{ccccl}
\hline 2006 & 2016 & Incremento & OCDE & Diferencia \\
\hline 461 & 496 & $7.05 \%$ & 493 & $0.6 \%$ por encima de la media \\
\hline
\end{tabular}

Estos datos evidencian una clarísima mejora de los resultados en Comprensión lectora (tabla I), posicionándose por encima de la media. Por competencia lectora entendemos la capacidad para comprender, utilizar, reflexionar e interesarse por los textos escritos, para alcanzar los propios objetivos, desarrollar conocimiento y potencial personal y participar en la sociedad (OECD, 2006). Probablemente una hipótesis a analizar para el incremento de estos resultados sea el impacto de la aparición de la Ley Orgánica de Educación (LOE, 2006) y su fuerte apuesta por la competencia lingüística y comunicativa, así como la aparición y fomento de programas de animación lectora. De igual forma, es innegable su apuesta por el desarrollo de la competencia digital. Dicho cambio favorece el aumento de la capacidad de leer en formato digital del alumnado, aspecto metodológico que se evalúa también en las pruebas PISA.

Al igual que ocurre con la Competencia lectora, se observa una mejora significativa en la competencia matemática, aunque sin llegar a posicionarnos por encima de la media europea (ver tabla II). La competencia matemática se define como la capacidad de reconocer y formular problemas matemáticos en situaciones diversas (OECD, 2006). La mejora de estos resultados se puede atribuir a la aparición de metodologías activas en la enseñanza de las matemáticas en los últimos años. Sin embargo, tanto la competencia matemática como la competencia en ciencias (ver tabla III), aunque aumentan en sus resultados de logro, se mantienen en la media (en Ciencias) o por debajo de la media (en Matemáticas).

Tabla II. Resultados en Matemáticas (2006-2016)

\begin{tabular}{ccccl}
\hline 2006 & 2016 & Incremento & OCDE & Diferencia \\
\hline 480 & 486 & $1.23 \%$ & 490 & $-.81 \%$ Por debajo de la media \\
\hline
\end{tabular}

Tabla III. Resultados en Ciencias (2006-2016)

\begin{tabular}{ccccl}
\hline 2006 & 2016 & Incremento & OCDE & Diferencia \\
\hline 488 & 493 & $1.01 \%$ & 493 & En la media europea \\
\hline
\end{tabular}

Estos resultados varían en función de las comunidades autónomas, presumiblemente por no tener un sistema educativo centralizado que facilite los mismos recursos y políticas entre comunidades. Valdría la pena analizar, en estudios longitudinales a escala nacional, los factores que permiten conocer en 
profundidad la causa de las diferencias presentadas en estos resultados. Por otra parte, no se puede ignorar cómo la desigualdad en el reparto de recursos económicos entre comunidades afecta de forma evidente la equidad y competitividad de los alumnos españoles frente a los de otros países europeos (Ferrer et al., 2010).

Al respecto, Schleicher (2016) afirma que un aspecto a tener en cuenta en el análisis del impacto de los resultados del informe es la urgente necesidad de invertir en calidad y no sólo en cantidad de recursos. El informe Focus (OCDE, 2017) evidencia el decrecimiento, en los últimos años, de la equidad de recursos en España con relación a otros países y comunidades autónomas (figura 1). Este decrecimiento obedece a que las políticas educativas se han enfocado en mejorar los resultados de los alumnos excelentes.

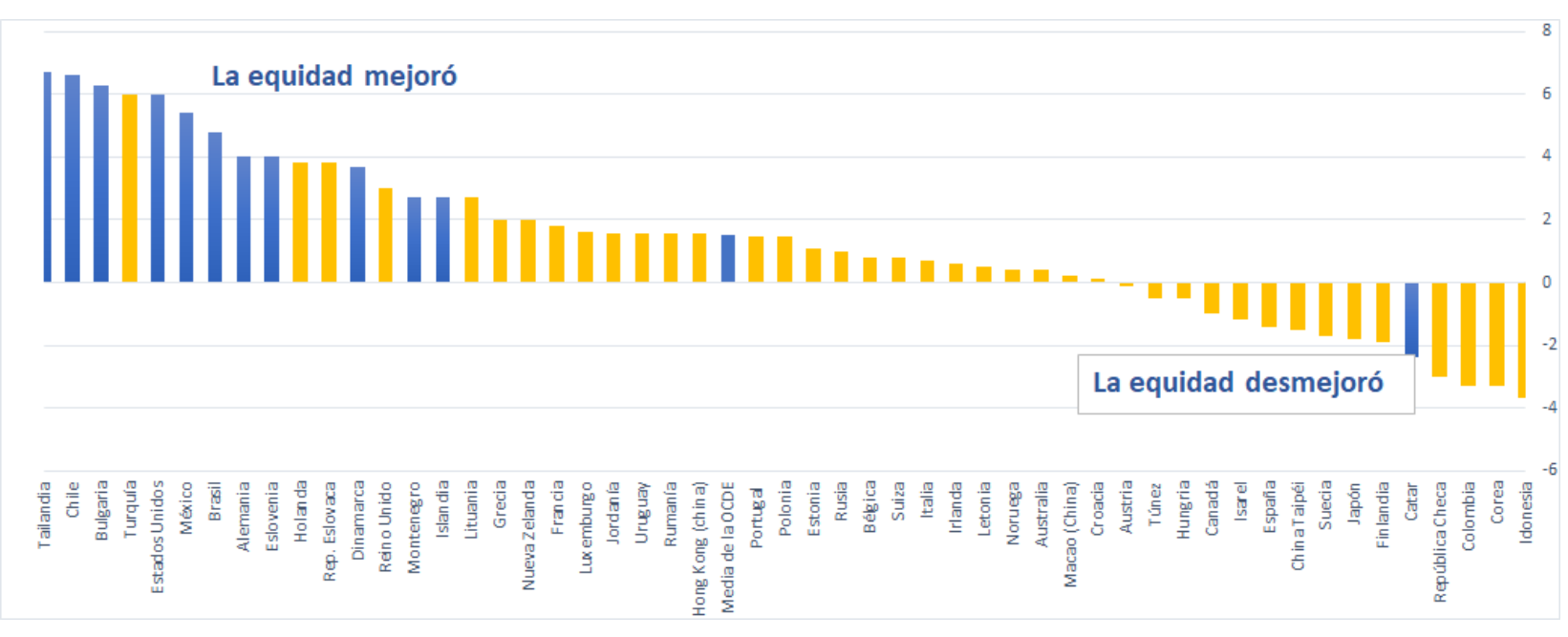

Figura 1. Tendencias en la mejora de la equidad en los países participantes en PISA (OCDE, 2017)

Así pues, sería interesante analizar el nivel de inversión en costes y tiempo, así como, la preparación de la participación de España en PISA, para determinar con mayor rigor la repercusión por coste (rentabilidad) que supone no sólo la participación en el mismo, sino lo más importante, el aprovechamiento del impacto en la toma de decisiones político-educativas.

Por ejemplo, sabemos que España invierte mayor número de horas lectivas en educación Primaria y Secundaria que los demás países de Europa y, sin embargo, la inversión de tiempo no conlleva mejores resultados:

En Educación Primaria, el número de horas de instrucción al año en España es de 791 horas, situándose ligeramente por debajo de la media de la OCDE y por encima de la UE22. En la primera etapa de educación Secundaria, las diferencias entre España y los países de la OCDE y la UE22 son notables, ya que los alumnos españoles reciben 1044 horas anuales de clase, superando en 129 horas anuales la media de horas de clase en los países de la OCDE y en 149 horas a la media en los países de la UE22 (Ministerio de Educación Cultura y Deporte [MECD] 2016, p. 52).

Por otra parte, resulta llamativo cómo el Informe del MECD (2016) sobre los resultados de la OCDE manifiesta que la ratio profesor-alumno en España es similar a la media de la OCDE y ligeramente inferior al de los países de la Unión Europea:

El tamaño de la clase (número medio de alumnos por clase) es de 21 alumnos en educación Primaria en España, siendo el mismo número en la OCDE y de 20 en la UE22. En la primera etapa de educación Secundaria, el número de alumnos por clase se incrementa hasta los 25 alumnos en España, siendo de 23 y 21 alumnos por clase en la OCDE y en la UE22 respectivamente (p. 56). 
Sin embargo, países como Japón o Corea del Sur, que tienen mayor ratio profesor-alumno, han priorizado el salario del profesorado y la calidad de su formación (OECD, 2012) y presentan mejores resultados que el resto de países:

La reducción de tamaño de la clase no es, por sí sola, una garantía suficiente para mejorar la calidad de los sistemas educativos. Pero, ¿cuál es la repercusión en el rendimiento de los estudiantes? Además de la optimización de los recursos públicos, reducir el tamaño de las clases para aumentar el rendimiento del alumnado es un enfoque que ha sido probado, debatido y analizado durante varias décadas. El tamaño de la clase puede afectar a la cantidad de tiempo y atención que un profesor puede dar a los estudiantes de forma individualizada, así como a las dinámicas sociales entre los estudiantes. Sin embargo, los resultados del Programa de la ocDE para la Evaluación Internacional de los Estudiantes (PISA) sugieren que los sistemas que priorizan la calidad del profesorado sobre las clases más pequeñas tienden a obtener mejores resultados... (p. 50).

\subsection{Costes e inversión de PISA y resultados}

Diversos investigadores manifiestan que la inversión en la participación en las pruebas PISA debería justificarse bajo la proyección a futuro de crecimiento en producción intelectual de cada país. En el estudio de Hanushek y Woessmann (2017) se presentan datos estadísticos que permiten identificar históricamente la relación entre mejora en habilidades cognitivas y crecimiento económico de los países. El análisis de estas variables permite identificar los beneficios de las reformas educativas en los países participantes. Así, al analizar estas dos variables y su correlación se identifican los aspectos que deberían, en el tiempo, justificar los resultados de las pruebas PISA. De igual manera, pone de manifiesto cómo la no trasferencia de los resultados tiene un alto coste para los países que no poseen un plan de aplicación de los mismos.

En este sentido, la OCDE (Hanushek y Woessmann, 2017) hace una proyección de lo que podría pasar con los países con mejores o peores resultados en PISA, en relación con la inversión económica y ganancia para el capital humano. Este estudio propone tres escenarios de participación para el análisis de los resultados: Polonia, que es uno de los países que presenta una baja infraestructura, ha mostrado un aumento significativo en PISA entre 2000 y 2006, aumentando en 29 puntos sus resultados $(0.25 \%$ de desviación estándar). Un segundo análisis toma como referencia a Finlandia, país con el mejor promedio de mejora y resultado a lo largo de su participación. Y, por último, el tercer escenario se proyectaría para los países cuyo resultado se ubique entre los de logro más bajo, por debajo de 400 puntos en PISA (con una desviación estándar por debajo del promedio de la OCDE).

Con respecto al primer escenario, Polonia, de continuar con esta proyección (Hanushek y Woessmann, 2017), cuando los estudiantes de mayor rendimiento empiecen a ser más significativos en el mercado laboral,su PIB aumentaría en 2042 en torno a un 3\% por encima de las previsiones. Esta proyección económica es mucho más importante cuando se tiene en cuenta que estas mejoras en la economía son dinámicas, ya que producen ganancias constantes, que generan ganancias anuales.

En el segundo escenario, Finlandia, es menos significativo, ya que los países con niveles altos no presentarían cambios drásticos en relación con su economía, ya que se retroalimenta y mantiene. Ello obedece a que los cambios son proporcionales a los logros presentados. La proyección de costes en tiempo e inversión económica (ver tabla IV) de la equiparación con los resultados de Finlandia (promedio de 546 puntos en PISA) a otros países supondría: 
Tabla IV. Equiparación de sistemas educativos europeos en relación con el promedio de Finlandia (2010)

\begin{tabular}{lcccc}
\hline $\begin{array}{c}\text { País } \\
\text { participante } \\
\text { en PISA }\end{array}$ & $\begin{array}{c}\text { Valor de la } \\
\text { reforma* }\end{array}$ & $\begin{array}{c}\text { Porcentaje } \\
\text { de PIB actual }\end{array}$ & $\begin{array}{c}\text { Aumento } \\
\text { de puntos } \\
\text { porcentuales }\end{array}$ & $\begin{array}{c}\text { Aumento } \\
\text { de puntos } \\
\text { en PISA }\end{array}$ \\
\hline España & 11.289 & $728 \%$ & 1.07 & 61.7 \\
\hline Finlandia & 0 & 0 & 0 & 0 \\
Francia & 10.424 & $461 \%$ & 0.72 & 41.3 \\
Italia & 18.094 & $927 \%$ & 1.31 & 75.6 \\
\hline Reino Unido & 7.326 & $307 \%$ & 0.49 & 28.5 \\
\hline
\end{tabular}

Nota: *En millones de dólares

Por tanto, la participación de los países supone una mirada a largo plazo, especialmente en relación con los resultados de futuro. Los objetivos más agresivos, como lograr que todos los estudiantes tengan un nivel de competencia mínima para la OCDE (una puntuación PISA de 400), implicarían incrementos agregados del PIB de cada país de alrededor de 200 billones de dólares (Hanushek y Woessmann, 2015). De igual manera, en el estudio proyectivo, según las relaciones de crecimiento históricas, el acercamiento de todos los países al sistema educativo de mejor desempeño de la OCDE en PISA (Finlandia), daría lugar a ganancias del orden de 260 billones de dólares (en un plazo de entre 20 a 90 años). En cualquier caso, los análisis manifiestan que es la calidad de los resultados de aprendizaje, no la duración de la escolarización, lo que marca la diferencia. Por ello, los resultados suponen una profunda reflexión en torno a los costes a todo nivel. A continuación, una reflexión sobre si es conveniente el alto precio en otros tantos aspectos, no sólo el económico, en relación con los resultados PISA.

\section{El alto precio de un sistema educativo orientado a PISA}

Podemos coincidir en que es positivo y necesario mejorar los resultados en los estándares de aprendizaje, aunque primero se deberían discutir cuáles son esos estándares, por qué deben ser esos y no otros, y quién debe decidir esas cuestiones.

Nadie se atrevería a afirmar que es negativo que aumente la calidad de la educación, ni que es malo que aumenten los resultados de los jóvenes en diferentes evaluaciones, aunque cada día son más los autores que se plantean al servicio de qué y de quién está esa llamada calidad de la educación, y si debemos evaluar realmente lo que estos programas evalúan.

En esa línea, también hay autores que analizan las consecuencias de un sistema educativo demasiado pendiente de los resultados y de un profesorado que está transformando su rol educativo, sus funciones, su formación, su estatus y reconocimiento social a causa de un proceso de enseñanza-aprendizaje demasiado orientado a la rendición de cuentas. Es, por ello, que nos planteamos si todo esto, realmente, nos sale a cuenta. La unESCO (Bokova, 2015) establece la dicotomía entre una educación con poder emancipador, con utilidad práctica para enfrentarse a la vida, que fomenta la capacidad crítica y favorece la sostenibilidad económica, social y medioambiental frente a una educación en la que se acumulan saberes sin ocuparse de su empleo y utilidad, en la que el saber sólo tiene por objetivo superar diferentes pruebas académicas o está orientada a satisfacer al mercado (Sanz et al., 2018).

Hemos analizado algunas medidas recogidas en la LOMCE destinadas a aumentar los resultados en PISA y en cómo dichos resultados han sido utilizados, tanto por medios de comunicación como por representantes políticos, como arma arrojadiza, instrumento de descrédito del sistema educativo y de erosión de los gobiernos en turno. Pero, sin darnos cuenta, estas luchas ideológico-políticas tienen consecuencias en la estructura, finalidad y organización del sistema educativo y de su personal docente. Todo ello, aun siendo conscientes de que los resultados en lectura, matemáticas y ciencias han ido aumentando de forma progresiva.

Y es esa la razón por la que ponemos en la balanza los pros y los contra y analizamos si realmente valió la pena. Hargreaves y Fink (2008) describen una relación de efectos dañinos para la educación a causa de la estandarización educativa: 
Restringió el currículum y destruyó la creatividad en el aula; limitó en las escuelas innovadoras su capacidad de conectar el aprendizaje con la vida de los alumnos; ensanchó la brecha de aprendizaje entre los centros educativos de élite y los demás; fomentó las estrategias impúdicas y calculadas para subir las puntuaciones en los test; socavó la confianza y la competencia del profesor; erosionó a los docentes, preocupados por cumplir con la fatigosa exigencia del currículum y los exámenes; precipitó la aparición de mayores índices de estrés, dimisiones y abandono; y amplió la resistencia al cambio entre los profesores. (p. 23).

\subsection{Consecuencias}

La educación, como recoge la Declaración Universal de los Derechos Humanos (1948) y la Constitución (1978), tiene por objeto el pleno desarrollo de la personalidad humana en el respeto a los principios democráticos de convivencia y a los derechos y libertades fundamentales. Esta visión humanista, donde el sentido último es la formación de la persona (Sanz y Serrano, 2016), se enfrenta con la corriente imperante en nuestro sistema educativo actual, donde el foco se sitúa en la defensa de ciertos valores económicos, de mercado, y que ha propiciado la aparición de nuevos conceptos: competencias, eficacia, rendición de cuentas, rentabilidad, indicadores de calidad, etc., en definitiva, la educación ha virado hacia posiciones de talante mercantilista. Lo que Torres-Santomé (2011, p. 94) definió como una "economización de las políticas educativas". Y esta es una primera consecuencia de la cual se derivan otras; Pring (2016) eleva, en esa misma línea, una crítica:

[Hacia aquellos que] transforman el lenguaje de los valores en algo medible, quienes reducen las aspiraciones educativas a una serie de objetivos observables, quienes consideran la educación exclusivamente como una suma de medios para alcanzar fines ulteriores no estrictamente educativos -tener buenas calificaciones, conseguir un empleo mejor, cumplir las expectativas del mercado, elevar la posición del centro educativo en el ranking nacional de escuelas... (p. 15).

La crisis económica, derivada de la crisis del petróleo de 1973, provocó el aumento de la inflación y la reducción de la actividad económica en muchos países de la Unión Europea y en Estados Unidos, y removió los cimientos del Estado del Bienestar. Además, generó en la conciencia social cierto pesimismo sobre la capacidad de la educación para transformar y mejorar la sociedad y la vida de sus ciudadanos (Hargreaves y Shirley, 2012).

Este cuestionamiento de la capacidad regeneradora de la educación hizo que los gobiernos tomasen el control de los sistemas educativos y los pusieran al servicio del mercado, llegando a considerar, como dicen Hargreaves y Fullan (2014, p. 216), "la enseñanza como una inversión a corto plazo del capital empresarial y [a] financiar el presente hipotecando el futuro de nuestros hijos." Ante esto, señalan, "podemos hacer de la enseñanza una inversión sostenible para el capital profesional y dar origen a un mundo de ganancias felices en el futuro."

Pero eso no se hizo; algunos gobiernos, a través de las medidas aprobadas en las diferentes leyes fueron centralizando las políticas educativas, dotando de mayor prescripción a los currículums, limitando su flexibilidad, así como la capacidad de los docentes para introducir innovaciones. Los objetivos académicos se fueron estandarizando y los contenidos curriculares se jerarquizaron en función de la lógica del mercado, pero curiosamente con parámetros más cercanos a la sociedad industrial que a los de la sociedad del conocimiento en la que vivimos. Valores como la creatividad, la invención y la resiliencia, o capacidades como la adaptación a contextos diversos, la resolución de conflictos y la inteligencia emocional, entre otros, han perdido peso entre los contenidos académicos, para centrarse en el desarrollo de las competencias matemáticas, lingüísticas y científicas. Por tanto, otra consecuencia es un currículum más rígido, mayor control político de la educación -a través de pruebas estandarizadas- y jerarquización de ciertas asignaturas, contenidos y competencias. 
Además, un proceso de enseñanza-aprendizaje enfocado a la preparación, al entrenamiento del alumno para mejorar los resultados en las diferentes pruebas, favorece la implementación de una metodología docente mecanicista, transmisora, memorística y repetitiva. Por lo que otra consecuencia es la ausencia de innovación.

Las diferentes pruebas de evaluación y los rankings tienen consecuencias para el sistema educativo. La competitividad entre escuelas provoca, por un lado, que se abra una brecha social interescolar en la que podemos distinguir escuelas "de primera" y "de segunda," donde los grupos con menos recursos (inmigrantes, personas con discapacidad, etc.) conforman el grueso de dichas escuelas.

Fullan (2002) exponía, en un sentido radicalmente opuesto, que "la pobreza, sobre todo entre los niños y las mujeres, el racismo, el consumo de drogas y otros graves problemas sociales y personales hacen que, día a día, la propuesta de equidad y excelencia sea más grave y conmovedora" (p. 23). Por ello, aboga por una educación con propósito moral. Por tanto, afirma que un sistema educativo que desvirtúa los resultados de PISA, tomando como referencia sólo los resultados de los alumnos más aventajados, donde la educación se convierte en mercancía y los alumnos son clientes, también tiene como consecuencia una visión negativa de la atención a la diversidad, que se opone a una escuela compensadora de desigualdades, equitativa y que desarrolla capacidades de y para todos los alumnos.

\subsection{Consecuencias de un profesorado en constante "evaluación"}

El cuerpo docente también se ve afectado por las consecuencias de unas políticas educativas al servicio del mercado, obsesionadas por los resultados. Muchos docentes viven con angustia la publicación de los resultados PISA y opinan que la utilización de dichos resultados -a veces de manera malintencionada (Hargreaves y Shirley, 2012)- por los medios de comunicación y de muchos gobernantes, favorecen el descrédito y la desconfianza de la sociedad hacia su trabajo. Fullan (2002) afirma que la sociedad suspende a sus docentes en un doble sentido: por no conseguir mejores resultados entre el alumnado y por no ayudar a mejorar las condiciones pedagógicas que hagan posible esa mejora.

Esta circunstancia provoca desmotivación y falta de ilusión por la carrera docente, tal y como demuestran las sucesivas políticas de la OCDE por atraer a los mejores estudiantes hacia estudios de Magisterio (OCDE, 2004). Esta desmotivación también provoca jubilaciones anticipadas, síndrome burnout y abundantes bajas por depresión entre el profesorado (Rodríguez et al., 2017). Hargreaves y O’Connor (2018) afirman que este "sentimiento de derrota" se traduce en la creencia de que se está defraudando a los alumnos si no se les prepara para los exámenes, obviando que lo realmente importante sería generar aprendizaje significativo, creativo y competente para la vida (Perrenoud, 2012). También, estas políticas educativas sitúan en segundo plano la formación integral de los alumnos, alejando a las Humanidades del currículum y olvidando el desarrollo de ciertas capacidades (Nussbaum, 2016).

Por tanto, si los docentes dedican gran parte de su quehacer profesional a la preparación para los exámenes y al cumplimentado de papeleo (burocracia), derivado del intento de regular y controlar por la Administración, vemos cómo su autonomía, su "capital decisorio" (Hargreaves y Fullan, 2014), su tiempo de práctica reflexiva, etc., se reducen sensiblemente, convirtiéndose en técnicos de la educación: "Los profesores no se consideran ni expertos en la materia, ni transmisores de la cultura, ni quienes deben ayudar a desarrollar las potencialidades creativas de los niños: son meros técnicos". (Abbs, 2003, p. 4).

Esta visión reduccionista del docente, convertido en mero transmisor de información, en "dispensador de currículum" (Pring, 2016), merman su desarrollo profesional y su capacidad creativa e innovadora. Además, también limita su capacidad de decisión sobre la discusión y elaboración del currículum, al contrario de lo que ocurre en otros países, como Finlandia, donde el "capital decisorio" del profesorado y su autonomía son los aspectos más valorados por los propios docentes.

Por ello, son muchas e importantes las consecuencias para el profesorado de un sistema educativo "obsesionado" por la evaluación y sus resultados. Una sociedad que desconfía de su trabajo y que, en algunas ocasiones, no lo valora suficientemente (Day y Gu, 2015). Una administración que sobrecarga de 
tareas "no pedagógicas" su horario escolar y que constantemente evalúa su quehacer profesional. Un proceso de enseñanza-aprendizaje más preocupado por las notas que por el aprendizaje, que obliga a los docentes a dedicar muchas horas y esfuerzos a preparar para los exámenes, coartando la creatividad, la innovación, la autonomía y la capacidad de decisión sobre los propios aprendizajes. En definitiva, una profesión desmotivada, que pierde poco a poco la pasión por enseñar (Day, 2011).

\section{Discusión y conclusiones}

Creemos que PISA es "una forma un tanto camuflada de presionar a los Estados para reformular sus políticas educativas" (Torres-Santomé, 2011, p. 186). Sin embargo, el relato que presentan los medios de comunicación y la política partidista hace que construyamos alrededor de estos resultados un discurso de postverdad.

De esta manera, aspectos clave en la educación como la creatividad, la innovación, la atención a la diversidad, la educación en valores y el desarrollo de capacidades corren el riesgo de quedar supeditadas, marginadas, frente a un simple entrenamiento para superar exámenes. Como consecuencia, el currículum académico y el horario escolar priorizan ciertas asignaturas -Matemáticas, Lengua y Ciencias- en detrimento de otras, tanto en cantidad de horas como en franjas horarias.

Además, sitúa a los docentes en el ojo del huracán con cada publicación de los resultados PISA. Los análisis erróneos, sesgados o malintencionados cuestionan su trabajo, merman su autoridad y reconocimiento social y les someten a un proceso constante de rendición de cuentas. La equidad del sistema educativo se resquebraja con cada evaluación. La distancia entre algunos centros escolares de barrios marginales y de élite, así como entre diferentes comunidades autónomas se agranda, con las consiguientes consecuencias de fractura social.

Estas conclusiones ponen de manifiesto que la hipótesis de partida se confirma. El mal uso de los resultados PISA conduce a un proceso de mercantilización de la educación; los fines educativos viran desde una educación de carácter humanista (formar ciudadanos) hacia una educación de corte utilitarista (formar mano de obra eficiente); los docentes se convierten en simples técnicos de la educación y su motivación e identidad profesional disminuyen. Por ello, creemos que se puede afirmar que PISA favorece la deshumanización del proceso educativo y reclamamos la vuelta a una educación más centrada en la persona y en su desarrollo integral.

\section{Referencias}

Abbs, P. (2003). Against the flow: education, the arts and postmodern culture. Routledge.

Bokova, I. (2015). Replantear la educación. ¿Hacia un bien común mundial? unEsco.

Cárdenas, C. y Huertas, F. J. (2013). El informe PISA en España. Un análisis al detalle. Revista de Currículum y Formación del Profesorado, 17(2), 243-262.

http://digibug.ugr.es/bitstream/10481/30018/1/rev172COL2.pdf

Day, C. (2011). Pasión por enseñar. La identidad personal y profesional del docente y sus valores. Narcea.

Day, C. y Gu, Q. (2015). Educadores resilientes, escuelas resilientes. Construir y sostener la calidad educativa en tiempos difíciles. Narcea.

Ferrer, F., Valiente, O. y Castel, J. L. (2010). Los resultados PISA-2006 desde la perspectiva de las desigualdades educativas: la comparación entre Comunidades Autónomas en España. Revista Española de Pedagogía, 245, 23-48. https://revistadepedagogia.org/volume/lxviii/no-245/

Fullan, M. (2002). Las fuerzas del cambio. Explorando las profundidades de la reforma educativa. Akal. 
Gadamer, H. G. (1977). Verdad y método. Fundamentos de una hermenéutica filosófica. Sígueme.

Hanushek, E. A. y Woessmann, L. (2015). The knowledge capital of nations: education and the economics of growth. MIT Press.

Hanushek, E. A. y Woessmann, L. (2017). The high cost of low educational performance: the long-run economic impact of improving PISA outcomes. OECD.

Hargreaves, A. y Fink, D. (2008). El liderazgo sostenible. Siete principios para el liderazgo en centros educativos innovadores. Morata.

Hargreaves, A. y Fullan, M. (2014). Capital profesional. Morata.

Hargreaves, A. y O'Connor, M. T. (2018). Collaborative professionalism: when teaching together means learning for all. Corwin Press.

Hargreaves, A. y Shirley, D. (2012). La cuarta vía. El prometedor futuro del cambio educativo. Octaedro.

Instituto Nacional de Evaluación Educativa. (2016). Educainee. Boletín de Educación, nº 51. http://educalab.es/documents/10180/14984/educainee51.pdf/5701f0e2-f17a-43b5-bc55-680d638f9f37

Jornet, J. M. (2016). Análisis metodológico del Proyecto PISA como evaluación internacional. Relieve, 22(1). http://dx.doi.org/10.7203/relieve.22.1.8293

Ley 2/2006. Ley Orgánica de Educación, de 3 de mayo de 2006. Diario Oficial.

Ley 8/2013. Ley Orgánica para la Mejora de la Calidad Educativa, de 9 de diciembre de 2013. Diario Oficial.

Lundgren, U. P. (2013). PISA como instrumento político: La historia detrás de la creación del programa PISA. Revista de Currículum y Formación del Profesorado, 1(2), 15-29.

http://digibug.ugr.es/bitstream/handle/10481/30005/rev172ART1.pdf?sequence=1\&isAllowed=y

Luzón, A. y Torres, M. (2013). La presencia de PISA en la literatura científica y su tratamiento en la prensa diaria. Revista de Currículum y Formación del Profesorado, 17(2), 193-224.

http://digibug.ugr.es/bitstream/handle/10481/30016/rev172ART12.pdf?sequence=1\&isAllowed=y

Marchesi, A. y Martínez, R. (2006). Escuelas de éxito en España. Sugerencia e interrogantes a partir del Informe PISA 2003. Santillana.

Martínez-Rizo, F. (2016). Impacto de las pruebas en gran escala en contextos de débil tradición técnica: experiencia de México y el Grupo Iberoamericano de PISA. Relieve, 22(1).

http://dx.doi.org/10.7203/relieve.22.1.8244

Ministerio de Educación Cultura y Deporte. (2016). Panorama de la educación, indicadores de la OCDE (Informe Español). Subdirección General de Documentación y Publicaciones.

Nussbaum, M. (2016). Not for profit. Why democracy needs the humanities. Princenton University Press.

OCDE (1991). Escuelas y calidad de la enseñanza. Informe internacional. Paidós.

OCDE (2004). La cuestión del profesorado: atraer, capacitar y conservar a profesores eficientes. Resumen en español. http://www.educacionyfp.gob.es/va/dam/jcr:6a3fbd74-e5c4-4a07-b144-6e694142f771/ xxieccee06ocdelacuestindelprofesorado-pdf.pdf 
OCDE (2015). PISA 2015. Assessment and analytical framework. Science, reading, mathematic and financial literacy. https://read.oecd-ilibrary.org/education/pisa-2015-assessment-and-analytical-framework/pisa2015-financial-literacy-framework 9789264281820-6-en\#page1

OCDE (2017). PISA In Focus 68. Autor. https://www.oecd-ilibrary.org/education/where-did-equity-ineducation-improve-over-the-past-decade 33602e45-en

OECD (2006). Assessing Scientific, Reading and Mathematical Literacy. A Framework for PISA 2006. Autor. https://www.oecd.org/pisa/39732471.pdf

OECD (2012). PISA 2012. Assessment and analytical framework. Mathematics, Reading, Science, problem solving and financial literacy. Autor.

https://www.oecd.org/pisa/pisaproducts/PISA\%202012\%20framework\%20e-book final.pdf

Perrenoud, P. (2012). Cuando la escuela pretende preparar para la vida. ¿Desarrollar competencias o enseñar otros saberes? Graó.

Pring, R. (2016). Una filosofía de la educación políticamente incómoda. Narcea.

Puelles, M. (2009). Calidad, reformas sociales y equidad social. En M. A. Santos (Ed.), Políticas educativas y compromiso social (pp. 25-39). Octaedro.

Rodríguez, J. A., Guevara, A. y Viramontes, E. (2017). Síndrome de burnout en docentes. Revista de Investigación Educativa de la REDIECH, 14, 45-67.

http://www.rediech.org/ojs/2017/index.php/ie rie rediech/article/view/39

Sanz, R. y Serrano, A. (2016). El desarrollo de capacidades en la educación. Una cuestión de Justicia Social. Sinéctica. Revista Electrónica de Educación, 46, 23-38.

https://sinectica.iteso.mx/index.php/SINECTICA/article/view/597

Sanz, R., Peris, J. A. y Escámez, J. (2018). The capabilities approach and values of sustainability: towards and inclusive Pedagogy. Journal of Innovation \& Knowledge, 3, 76-81.

https://doi.org/10.1016/i.jik.2017.12.008

Schleicher, A. (2016). Teaching excellence through professional learning and policy reform: lessons from around the world, international summit on the teaching profession. OECD.

http://dx.doi.org/10.1787/9789264252059-en

Sociedad Española de Pedagogía. (1988). La calidad de los centros educativos. Caja Ahorros Alicante.

Tiana, A. (2009a). ¿Puede ser el informe PISA un referente válido para valorar la equidad y la calidad de la educación en España? En M. A. Santos (Ed.), Políticas educativas y compromiso social (pp. 41-58). Octaedro.

Tiana, A. (2009b). Por qué hicimos la Ley Orgánica de Educación. Wolters Kluwer.

Torres Santomé, J. (2011). La justicia curricular. El caballo de Troya de la cultura escolar. Morata.

Turner, R. (2006). El Programa Internacional para la Evaluación de los Alumnos (PISA). Una perspectiva general. Revista de Educación, Número extraordinario, 45-74.

http://www.educacionyfp.gob.es/revista-de-educacion/inicio.html 